University of Wollongong

Research Online

Faculty of Engineering and Information

Faculty of Engineering and Information

Sciences - Papers: Part A

Sciences

$1-1-2014$

\title{
Measuring Young's modulus using a self-mixing laser diode
}

Ke Lin

University of Wollongong, kl740@uowmail.edu.au

Yanguang Yu

University of Wollongong, yanguang@uow.edu.au

Jiangtao Xi

University of Wollongong, jiangtao@uow.edu.au

Yuanlong Fan

University of Wollongong, yf555@uowmail.edu.au

Huijun Li

University of Wollongong, huijun@uow.edu.au

Follow this and additional works at: https://ro.uow.edu.au/eispapers

Part of the Engineering Commons, and the Science and Technology Studies Commons

Research Online is the open access institutional repository for the University of Wollongong. For further information contact the UOW Library: research-pubs@uow.edu.au 


\title{
Measuring Young's modulus using a self-mixing laser diode
}

\begin{abstract}
This paper presents a novel approach for determining the Youngâ $\mathrm{E}^{\mathrm{T} \mathrm{w}} \mathrm{s}$ modulus by using a self-mixing laser diode (SMLD). An SMLD system consists of a laser diode (LD), a microlens and an external target. With a small portion of light backscatterd or reflected by the target re-entering the LD inside cavity, both the amplitude and frequency of the LD power are modulated. This modulated LD power is referred as a self-mixing signal (SMS) which is detected by the photodiode (PD) packaged in the rear of the LD. The external target is the tested sample which is in damping vibration excited by a singular elastic strike with an impulse tool. The vibration information from the tested sample is carried in the SMS. Advanced data processing in frequency-domain is applied on the SMS, from which the resonant frequency of the vibration can be retrieved, and hence Youngâ $€^{\mathrm{TM}} \mathrm{s}$ modulus is calculated. The proposed method has been verified by simulations. 2014 SPIE.
\end{abstract}

\section{Keywords}

modulus, self, mixing, measuring, laser, diode, young

Disciplines

Engineering | Science and Technology Studies

\section{Publication Details}

K. Lin, Y. Yu, J. Xi, Y. Fan \& H. Li, "Measuring Young's modulus using a self-mixing laser diode," in Proceedings of SPIE - The International Society for Optical Engineering, 2014, pp. 89750B-1-89750B-8. 


\title{
Measuring Young's Modulus Using a Self-Mixing Laser Diode
}

\author{
Ke Lin ${ }^{1}$, Yanguang $\mathrm{Yu}^{* 1}$, Jiangtao $\mathrm{Xi}^{1}$, Yuanlong Fan ${ }^{1}$ and Huijun $\mathrm{Li}^{2}$ \\ ${ }^{1}$ School of Electrical, Computer and Telecommunications Engineering, University of Wollongong, \\ Northfields Avenue, Wollongong, NSW, 2522, Australia \\ ${ }^{2}$ School of Mechanical, Materials and Mechatronic, University of Wollongong, Northfields Avenue, \\ Wollongong, NSW, 2522, Australia
}

\begin{abstract}
This paper presents a novel approach for determining the Young's modulus by using a self-mixing laser diode (SMLD). An SMLD system consists of a laser diode (LD), a microlens and an external target. With a small portion of light backscatterd or reflected by the target re-entering the LD inside cavity, both the amplitude and frequency of the LD power are modulated. This modulated LD power is referred as a self-mixing signal (SMS) which is detected by the photodiode (PD) packaged in the rear of the LD. The external target is the tested sample which is in damping vibration excited by a singular elastic strike with an impulse tool. The vibration information from the tested sample is carried in the SMS. Advanced data processing in frequency-domain is applied on the SMS, from which the resonant frequency of the vibration can be retrieved, and hence Young's modulus is calculated. The proposed method has been verified by simulations.
\end{abstract}

Keywords: Young's modulus, laser diode, self-mixing interference

\section{INTRODUCTION}

Young's modulus is one of fundamental mechanical parameters for describing various kinds of materials' behaviour, such as porosity, texture and intergranular phases ${ }^{1}$. Therefore, it is of significant interest to know the value of this parameter since knowledge of Young's modulus is required both for analysis and design. Significant amount of methods have been devoted to determining Young's modulus experimentally, which are categorized as static ${ }^{2,3}$ and dynamic ${ }^{4-6}$.

Static methods for Young's modulus measurement mainly refer to as tensile or compressive tests, which are based on direct measurements of stresses and strains during mechanical tests. However, there are many practical difficulties associated with achieving a straight portion at the beginning of the stress-strain curve. Besides, they are inadequate when dealing with brittle materials such as ceramics, whose modulus is notoriously hard to measure. Because to reach a measurable deformation a comparatively high stress will be applied, thus resulting in passive repercussion to the structure of the material and creating micro-cracks in these materials ${ }^{7}$. Other afterwards derivative static methods, such as four-bending test or indentation method, are either impossible for a continuous measurement or sensitive to undesirable disturbance ${ }^{8}$. Though static test equipment is readily available, it still requires specialized workman to perform the test. So, static methods are neither feasible nor realistic to carry them in a cost effective way in a high through-put computer controlled test.

Dynamic methods were first developed in the United States in the early 1930s, which are tests based on resonant frequency extraction for modulus measurement, since for a vibrating beam of given dimensions, the resonant frequency is mainly related to its dynamic elastic modulus. Dynamic methods present to be a more superior technique than static

* yanguang@uow.edu.au; phone:+61242218187 
method due to its ease of specimen preparation, wide variety of specimen shapes and sizes, great precision, and applicability over a wide temperature range. One of the simplest dynamic ways is based on the so-called Impulse Excitation Technique (IET) in which an impulse tool is used to generate a singular strike on proper position of either a rectangular or a circular specimen that is ready to be tested. The dimension of the specimen and support position, as well as the impact locations are considered to induce flexural or torsional modes of vibration. The vibration signal will be detected using a non-contact microphone or a contact piezoelectric transducer and analysed by a signal processor to determine the resonant frequency ${ }^{8-10}$. However, many results of current IET methods still remain vulnerable to outside conditions ${ }^{11}$.

Laser interferometry is a well-established technique, widely used in the industrial and laboratory environments to measure velocity, vibration, displacement and distance, and its sensitivity is very high (which can reach to sub-nano metres) ${ }^{12}$. In order to accurately measure the Young's modulus, this paper proposes a novel non-contact and nondestructive measurement technique based on dynamic IET using a self-mixing laser diode. The paper aims to retrieve the resonant frequency of tested specimen from SMS signals and from which to calculate the Young's modulus. Lower mode shapes of the vibrating specimen are studied. The factors which may degrade the measurement accuracy are analysed.

\section{PRINCIPLE OF MEASUREMENT}

The self-mixing laser diode (SMLD) system mainly consists of a laser diode (LD), a microlens and an external target as shown in Figure 1. The LD is biased with a dc current through the LD controller. The temperature of LD is maintained at $25 \square \pm 0.1 \square$ by the temperature controller. A lens is used to focus the emitted laser. The test-piece sample is recognized as the external target. When the sample is set into vibration, a small portion of light will be backscatterd or reflected by the target and re-enter the LD internal cavity. Both the amplitude and frequency of the LD power are modulated. This modulated LD power is referred to as a self-mixing signal (SMS) which is detected by the photodiode (PD) packaged in the rear of the LD and amplified by a trans-impedance amplifier, then finally acquired by personal computer through $\mathrm{A} / \mathrm{D}$ converters.

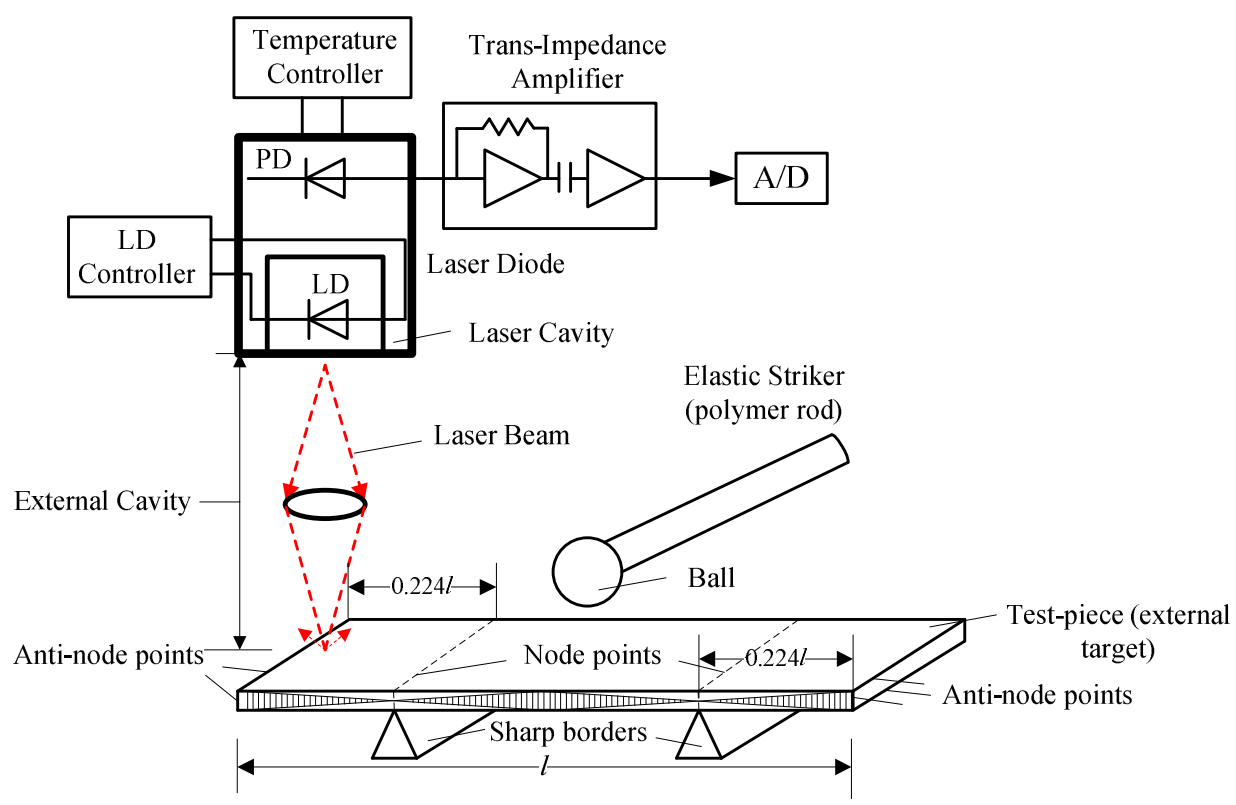

Figure 1. Schematic diagram for measuring Young's modulus using an SMLD system. 
The test-piece used as the external target is made of steel $\left(92 * 10 * 4 \mathrm{~mm}^{3}\right)$ and put onto two sharp borders. From these borders, the two ends of the test-piece maintain free and the distance between the sharp border and the extreme of the test-piece is 0.224 times the total length $l$ of the test-piece, making it vibrate coupled with as few mode shapes of vibration as possible. To induce a vibration for the test-piece, a small steel ball ( $5 \mathrm{~mm}$ in diameter) glued to the end of a flexible 10-cm long polymer rod is forced to impact exactly at the centre of the sample, whose vibrational information is therewith picked up by the LD. Care should be taken to position the laser beam at the end of the test-piece as close as possible, since the test-piece is in flexural vibration, and the anti-node points are all gathered to both ends of the testpiece.

A widely accepted mathematical model for describing an SMLD system is presented below ${ }^{13}$ :

$$
\begin{gathered}
\phi_{0}(n)=4 \pi L(n) / \lambda_{0} \\
\phi_{F}(n)=\phi_{0}(n)-C \sin \left[\phi_{F}(n)+\arctan (\alpha)\right] \\
G(n)=\cos \left(\phi_{F}(n)\right) \\
P(n)=P_{0}[1+m G(n)]
\end{gathered}
$$

where $n$ is the discrete time index; $\phi_{0}(n)$ and $\phi_{F}(n)$ are the laser phase with and without feedback respectively and $L(n)$ is the length of the external cavity, i.e., the perpendicular distance from the facet of the laser to the external test-piece; $\lambda_{0}$ is the wavelength of the emitted laser without feedback; $\alpha$ is the Linewidth Enhancement Factor (LEF); $C$ is the optical feedback level factor. $P_{0}$ is the intensity of the LD without external cavity; $m$ is modulation index for the laser intensity. When using a singular elastic striker to properly hit the centre of the specimen, the specimen would thus be set into damping vibration. Denoting the vibration by $y(n)$ (a function of the time), therefore, we have $L(n)=L_{0}+y(n)$, where $L_{0}$ is the distance between the laser facet and the equilibrium position of the vibration of the specimen. According to the above system model, the vibration $y(n)$ is carried in an SMS signal $G(n)$.

Figure 2. (b) shows an example of a practical SMS while the specimen is set into vibration. An SMS includes the ascending and the descending part which respectively corresponds to the specimen away from and close to the laser. Each fringe as indicated in Figure 2.(b) corresponds to $2 \pi$ phase change of SMS or $\lambda_{0} / 2$ change of the displacement of laser incidence point on the test-piece and the number decreases since the incident point on the test-point would vibrate in an attenuated form ${ }^{14}$.

The test-piece beam to be tested is termed as a free-free clamped one, since no forces or moments are applied at its ends during the test. In this paper, shear deformation is considered, so Timoshenko beam theory was adopted for modelling the beam's vibration, from which the mode shapes and resonant frequencies of the vibration could be obtained. 
(a)
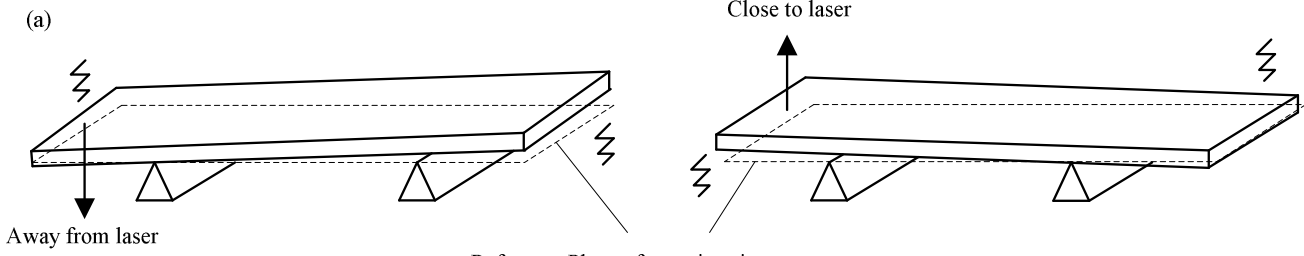

Reference Plane of test-piece in rest

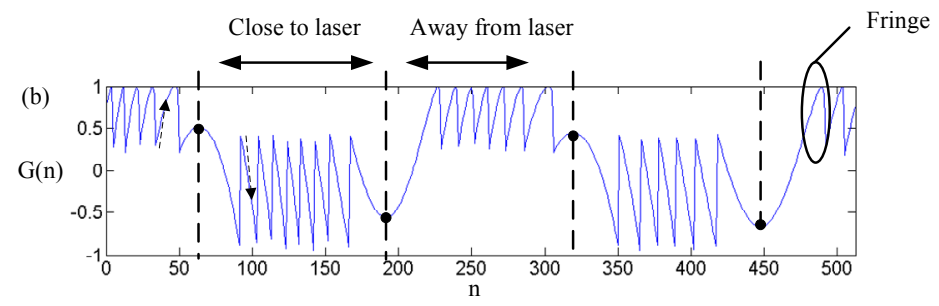

Figure 2. (a) Visualization of a free-free beam's vibration and (b) corresponding SMS with $\alpha=3, C=3$

The beam's vibration is governed by the equation below ${ }^{15}$.

$$
\rho A \frac{\partial^{2} w(x, n)}{\partial n^{2}}+\gamma \frac{\partial w(x, n)}{\partial n}+E I \frac{\partial^{4} w(x, n)}{\partial x^{4}}=0 \quad(0 \leq x \leq l)
$$

$E, I, A, \rho$ are respectively the Young Modulus, second moment of area of the cross section, density and cross section area of the beam. The damping term $\gamma \frac{\partial w(x, n)}{\partial n}$ models the internal energy loss mechanism of the beam. $w(x, n)$ is the displacement function of an arbitrary point with distance $x$ from the left end of the beam. $l$ is the length of the beam. The solution of Eq. (5) can be written as a standing wave, separating the spatial and temporal component.

$$
w(x, n)=V(x) y(n)
$$

Then detach the same variables into respective side of the equation. Since both sides of the equation are equal for all values of $x$ and $n$, which could be arbitrarily denoted as an constant $-\omega^{2}$. Thus the motion equation can be written as:

$$
\begin{gathered}
\frac{\partial^{4} V(x)}{\partial x^{4}}-\beta^{4} V(x)=0, \beta^{4}=\frac{\omega^{2}}{c^{2}}, c=\sqrt{\frac{E I}{\rho A}} \\
\frac{\partial^{2} y(n)}{\partial n^{2}}+\frac{\gamma}{\rho A} \frac{\partial y(n)}{\partial n}+\omega^{2} y(n)=0
\end{gathered}
$$

The general solution of the spatial and temporal part can be respectively written as:

$$
V_{i}(x)=\cosh \left[\left(\beta_{i} l\right) \frac{x}{l}\right]+\cos \left[\left(\beta_{i} l\right) \frac{x}{l}\right]-\sigma_{i}\left\{\sinh \left[\left(\beta_{i} l\right) \frac{x}{l}\right]+\sin \left[\left(\beta_{i} l\right) \frac{x}{l}\right]\right\}
$$




$$
\sigma_{i}=\frac{\cosh \left(\beta_{i} l\right)-\cos \left(\beta_{i} l\right)}{\sinh \left(\beta_{i} l\right)-\sin \left(\beta_{i} l\right)}
$$

and

$$
\begin{gathered}
y_{i}(n)=\sqrt{C_{1}^{\prime 2}+C_{2}^{\prime 2}} e^{-\zeta \omega_{i} n} \sin \left(\sqrt{1-\zeta^{2}} \omega_{i} n+\varphi_{0}\right), \\
\varphi_{0}=\tan ^{-1}\left(\frac{C_{1}^{\prime}}{C_{2}^{\prime}}\right), C_{1}^{\prime}=y_{0}, C_{2}^{\prime}=\frac{\dot{y}_{0}+\zeta \omega_{i} y_{0}}{\sqrt{1-\zeta^{2}} \omega_{i}}
\end{gathered}
$$

where $y_{0}$ and $\dot{y}_{0}$ is respectively the initial displacement and velocity of the arbitrary point on the beam. $\zeta$ is the viscous damping ratio of the environment. $i$ indicates the number of the mode shapes of vibration. The constant $\beta_{i} l$ above can be acquired through the application of boundary conditions, which include zero moment and zero shears at both ends of the beam. This requirement gives the characteristic equation. Table 1 shows the value of $\beta l$ under different mode shapes of vibration.

$$
\cos (\beta l) \cosh (\beta l)=1
$$

Table 1. Roots of the characteristic equation for a free-free beam.

\begin{tabular}{|c|c|}
\hline Root & Value \\
\hline $\boldsymbol{\beta}_{\mathbf{0}} \boldsymbol{l}$ & 0.00 \\
\hline $\boldsymbol{\beta}_{\mathbf{1}} \boldsymbol{l}$ & 4.73 \\
\hline $\boldsymbol{\beta}_{\mathbf{2}} \boldsymbol{l}$ & 7.85 \\
\hline $\boldsymbol{\beta}_{\mathbf{3}} \boldsymbol{l}$ & 11.00 \\
\hline $\boldsymbol{\beta}_{\mathbf{4}} \boldsymbol{l}$ & 14.14 \\
\hline $\boldsymbol{\beta}_{\mathbf{5}} \boldsymbol{l}$ & 17.28 \\
\hline $\boldsymbol{\beta}_{\boldsymbol{i}} \boldsymbol{l}$ & $(2 \mathrm{i}+1) \pi / 2, \mathrm{i}>5$ \\
\hline
\end{tabular}

Accordingly, from the Eq. (7), the analytical equation between the angular resonant frequency and the Young's modulus could be expressed by following.

$$
\begin{gathered}
\omega_{i}=\beta_{i}^{2} c=\frac{\left(\beta_{i} l\right)^{2}}{l^{2}} \sqrt{\frac{E I}{\rho A}} \\
E=\frac{l^{4} \rho A \omega_{i}}{\left(\beta_{i} l\right)^{4} I}
\end{gathered}
$$

Hence, Young's modulus can be readily determined from Eq. (15) as long as the resonant frequency can be obtained.

\section{DETERMINATION OF RESONANT FREQUENCY}

Figure 3 shows the overall design scheme of the algorithm for determining resonant frequency. When the test-piece beam is practically set into vibration, different external disturbance needs to be taken into consideration, such as the 
support location or different percussion on the beam with different force that probably would result in various coupling modes of vibration. Thus, after obtaining the SMS from the SMLD system, a phase unwapping technique is used for reconstructing $\phi_{0}(n)$ that corresponds to vibration displacement. During the deconvoluting process, the sensed SMS from one point of the vibrating beam will be used for a phase jump tracing and then the phase unwrapping. An optimal feedback level of the self-mixing interferometry will be obtained for reconstructing the phase after iterative optimization according to the relationship between the unwrapped phase, feedback level and the displacement ${ }^{16}$. Then, Fast Fourier Transformation will be used for retrieving the resonant frequency from the reconstructed $\phi_{0}(n)$ after observation of the error through comparing the reconstructed with the original $\phi_{0}(n)$.

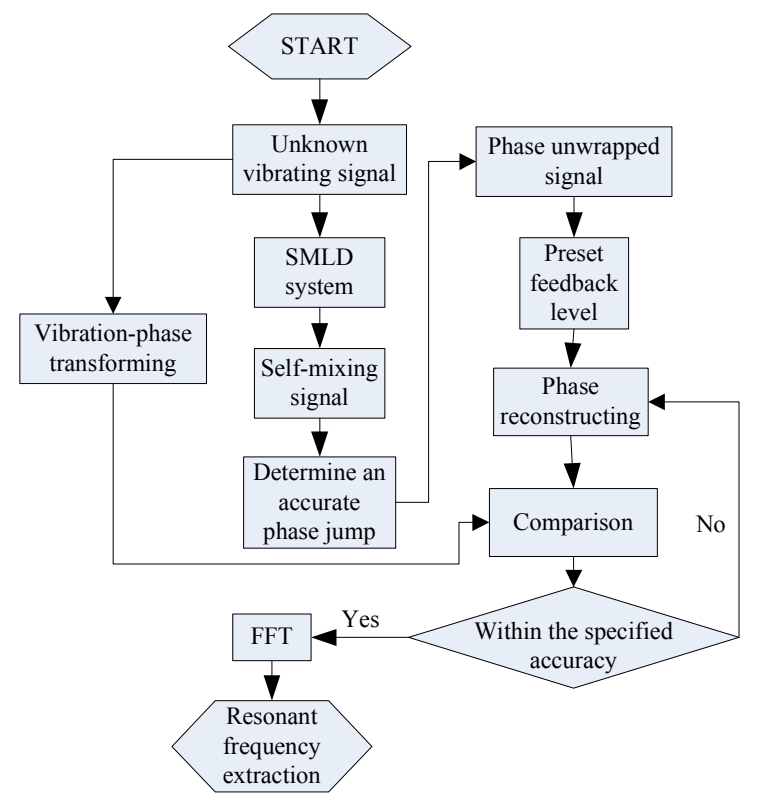

Figure 3. The design scheme based on SMLD for resonant frequency extraction

\section{SIMULATIONS AND RESULTS}

Equation (9) describes the $i$ th flexible mode shape of free-free beam's vibration. Each of mode shapes satisfies the spatial differential Eq. (7) and associated boundary conditions for the free-free beam. A free-free beam will execute only motions which are some combination of these mode shapes. Simulations on first four mode shapes for a free-free beam are shown in Figure 4.

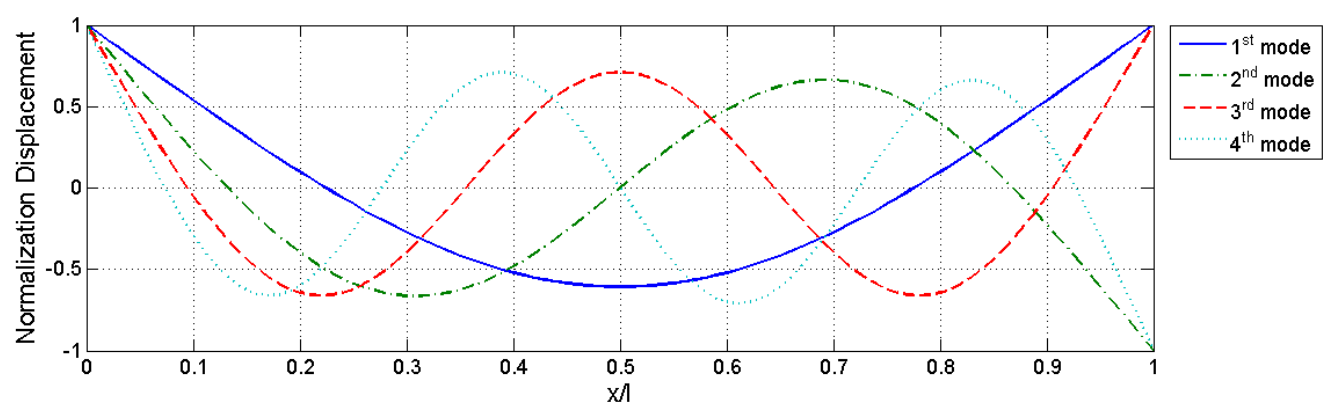

Figure 4. First 4 mode shapes of a free-free beam's vibration 
When the test-piece beam is vibrating under first 4 mode shapes coupled, the superposition of displacement profile of 4 different mode shapes is composed of the maximum displacement of each corresponding point on the beam. Then these corresponding points on the beam will be vertically vibrating in a damped sinusoidal form under 4 mode shapes coupled together. Figure 5 shows laser phase $\phi_{0}(n)$, i.e., the waveform of potential vibration on the anti-vibration points and corresponding SMS of the incidence point of the laser on the beam and reconstructed phase waveform for the first 4 mode shapes.
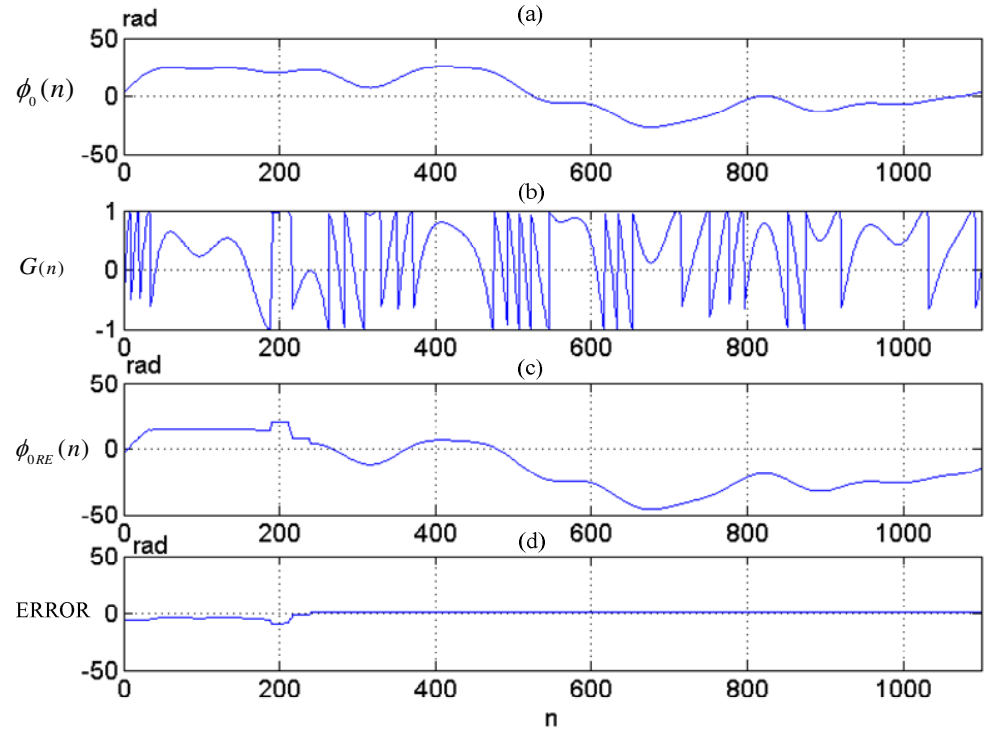

Figure 5. (a) Laser phase $\phi_{0}$ ( $n$ ) of potential vibration on the anti-vibration points, (b) corresponding SMS, (c) $\phi_{0 R E}(n)$, i.e., the reconstructed $\phi_{0}(n)$ and (d) the ERROR between reconstructed and original $\phi_{0}(n)$

Figure 5(d) shows the error between the reconstructed vibration phase waveform and the original. Figure 6 reveals the discrepancy of FFT results for original and reconstructed $\phi_{0}(n)$. The simulation results indicate that current algorithm for reconstructing vibration waveform could be smoothly applied for deconvoluting and reconstructing the vibration even under several coupling mode shapes. Though a micro error is found during the reconstruction, the deviation of extraction of the resonant frequency is nearly negligible. The spectrum of $\phi_{O R E}(n)$ is practically overlapped with the spectrum of $\phi_{0}(n)$. That is to say, the resonant frequency obtained from the reconstructed vibration wave form could be realistically viable for Young's modulus calculation in Eq. (15).

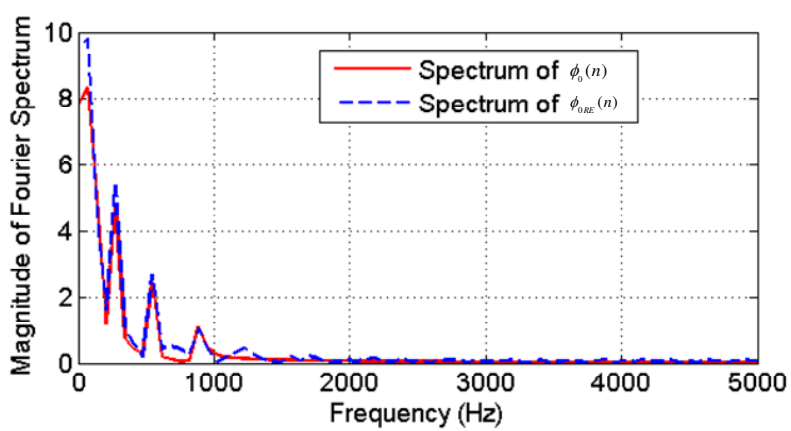

Figure 6. Comparison of spectrum of resonant frequency of reconstructed and original vibration waveform 


\section{CONLUSION}

A new technique for evaluation of the Young's modulus of a free-free beam sample is presented. The method utilizes the phenomenon of self-mixing interference to rebuild the original vibration signal based on the current reconstruction algorithm. After comparison of rebuilt phase waveform with the original one, the extraction of resonant frequency from two signals reasonably agreed with each other, which sufficiently prove the feasibility of this method for application of Young's modulus measurement in materials.

\section{REFERENCES}

[1] Radovic, M., Lara-Curzio, E., and Riester, L., "Comparison of different experimental techniques for determination of elastic properties of solids," Mat. Sci. Eng. A-Struct., 368(1): 56-70 (2004).

[2] Xiao, L. F., Duan, R., and Chen, X. G., "Study on the Electrometric Method to Measure the Young's Modulus of Metal Materials," Adv. Mat. Res., 652: 1319-1334 (2013).

[3] Zou, Q., Li, Z., and Liu, L., "New methods for measuring mechanical properties of thin films in micromachining: Beam pull-in voltage $\left(\mathrm{V}_{\mathrm{PI}}\right)$ method and long beam deflection (LBD) method," Sensor Actuat. A-Phys., 48(2): 137-143 (1995).

[4] Pritz, T., "Dynamic Young's modulus and loss factor of floor covering materials," Appl. Acoust., 49(2): 179190 (1996).

[5] Ding, X., Ko, W. H., and Mansour, J. M., "Residual stress and mechanical properties of boron-doped p+-silicon films," Sensor Actuat. A-Phys., 23(1): 866-871 (1990).

[6] Li, C. and Zhu, Z., "Dynamic Young's modulus of open-porosity titanium measured by the electromagnetic acoustic resonance method," J. Porous Mater., 13(1): 21-26 (2006).

[7] Lord, J. and Morrell, R., "Elastic modulus measurement-obtaining reliable data from the tensile test," Metrologia, 47(2): S41 (2010).

[8] Suansuwan, N. and Swain, M. V., "Determination of elastic properties of metal alloys and dental porcelains," J. Oral Rehabil., 28(2): 133-139 (2001).

[9] Salem, J. A. and Singh, A., "Polynomial expressions for estimating elastic constants from the resonance of circular plates," Mat. Sci. Eng. A-Struct., 422(1-2): 292-297 (2006).

[10] ASTM Standard C1259 - 08e1, "Standard Test Method for Dynamic Young's Modulus, Shear Modulus, and Poisson's Ratio for Advanced Ceramics by Impulse Excitation of Vibration," ASTM., West Conshohocken, PA (2009).

[11] Heritage, K., Frisby, C., and Wolfenden, A., "Impulse excitation technique for dynamic flexural measurements at moderate temperature," Rev. Sci. Instrum., 59: 973 (1988).

[12] Donati, S., "Developing self - mixing interferometry for instrumentation and measurements," Laser Photonics Rev., 6(3): 393-417 (2012).

[13] Xi, J., Yu, Y., Chicharo, J. F., and Bosch, T., "Estimating the parameters of semiconductor lasers based on weak optical feedback self-mixing interferometry," IEEE J. Quantum Electron., 41(8): 1058-1064 (2005).

[14] Giuliani, G., Norgia, M., Donati, S., and Bosch, T., "Laser diode self-mixing technique for sensing applications," J. Opt., 4(6): S283-S294 (2002).

[15] Coşkun, S. B., Atay, M. T., and Öztürk, B., "Transverse vibration analysis of euler-bernoulli beams using analytical approximate techniques," Advances in Vibration Analysis Research, InTech Publications, Vienna, Austria: 1-22 (2011).

[16] Fan, Y., Yu, Y., Xi, J., and Chicharo, J. F., "Improving the measurement performance for a self-mixing interferometry-based displacement sensing system," Appl. Opt., 50(26): 5064-5072 (2011). 